\title{
COMPARING THE EFFECT OF SECOND-GENERATION ANTIPSYCHOTICS VERSUS SELECTIVE SEROTONIN REUPTAKE INHIBITORS IN REFRACTORY OBSESSIVE-COMPULSIVE DISORDER: A SYSTEMATIC REVIEW OF THE PAST, PRESENT, AND FUTURE CLINICAL TRIALS
}

\author{
MEHDI SAYYAH $^{1}$, FAKHER RAHIM ${ }^{2}$ \\ ${ }^{1}$ Department of Psychiatry, Faculty Member of Education Development Center (EDC), Ahvaz Jundishapur University of Medical Sciences, \\ Ahvaz, Iran. ${ }^{2}$ Department of Molecular Medicine, Health Research Institute, Research Center of Thalassemia \& Hemoglobinopathies, \\ Ahvaz Jundishapur University of Medical Sciences, Ahvaz, Iran. Email: Bioinfo2003@gmail.com
} Received: 06 August 2016, Revised and Accepted: 03 October 2016

\section{ABSTRACT}

Objective: In this concise and systematic review, the trend of using major medication modalities prescribed for refractory obsessive-compulsive disorder (OCD), including serotonin-specific reuptake inhibitors (SSRIs) and second-generation antipsychotics (SGAs) are discussed.

Methods: We systematically searched PubMed and Cochrane Central Register of Controlled Trials (CENTRAL) systematically using Mesh terms. OCD is extremely disabling and associated with considerable depression and other serious psychiatric illnesses.

Results: Through databases, we found 78 randomized clinical trials (RCTs), which included selective SSRI compared with routine drug therapy or placebo. Out of these 78 studies, 62 studies were conducted on adult patients with OCD, comprising 7920 cases. While only 16 RCTs were performed on children and adolescents with OCD, including 1313 people. We found 24 clinical trial studies related to SGAs, of which were conducted on adult patients with OCD, including 992 cases.

Conclusion: As our data showed among the SSRIs, fluvoxamine has been particularly well studied and used in RCTs in both children and adolescents with OCD. According to the summary of our review, it will be better when therapists use SGAs in the early treatment programs of refractory OCD. Thus, considering our reviewed, it seems that the first choice of early treatment programs of refractory OCD is fluvoxamine in combination with quetiapine or aripiprazole.

Keywords: Obsessive-compulsive disorder, Refractory, Second-generation antipsychotic drugs, Selective serotonin reuptake inhibitors.

(c) 2017 The Authors. Published by Innovare Academic Sciences Pvt Ltd. This is an open access article under the CC BY license (http://creativecommons. org/licenses/by/4. 0/) DOI: http://dx.doi.org/10.22159/ajpcr.2017.v10i1.14528

\section{INTRODUCTION}

Obsessive-compulsive disorder (OCD) is a mental health condition with an unwanted, unpleasant thought, image, or urge that repeatedly enters a person's mind. OCD affects about $2-3 \%$ of people over the course of their lifetimes [1,2]. OCD is the result of common psychological social and genetic factors interaction [3]. In the biological factors could mention the serotonin disorder in the brain, which for the treatment of this aspect, drug treatment is recommended to set serotonin in the brain.

Drug treatment is one of the most common methods of treatment of acute agitation in patients with clinical mental health disorders. Antipsychotic drugs are, therefore, used in the acute treatment, chronic psychotic disorders, and other psychiatric conditions [4,5]. Firstgeneration antipsychotic medications (FGAs), which are also known as classical neuroleptic or traditional antipsychotics, which typically used to treat psychosis such as schizophrenia, acute mania, agitation, and other psychiatric conditions [6]. The FGAs act through blocking the dopamine (DAT) $\mathrm{D}_{2}$ neuro-receptor, which leads to the development of a subsequent series of new antipsychotics [7]. According to the potency of FGAs in binding to DAT $\mathrm{D}_{2}$ neuro-receptor, these drugs divided into two categories include low and high potency groups $[8,9]$. Some of the reported complications compose dyskinesia, hyperkinesia, and involuntary movements in the face and extremities [10]. Secondgeneration antipsychotic drugs (SGAs) that were introduced in 1989, which is also known as atypical antipsychotics are generally lower risk of extrapyramidal side effects compared with FGAs [10]. However, these drugs generally cause higher rates of weight gain and life-shortening metabolic disturbances, although side effects of any medication profile are different [11].
Selective serotonin reuptake inhibitors (SSRI), deal with neurochemical imbalance that is the key reason in mental health disorders $[12,13]$. People with acute mental health condition, suffer from a lack of serotonin in certain areas of the brain [14]. Serotonin is a chemical neurotransmitter that plays an important role in the mood regulation, is one of the key factors in lack of balance in mood disorders such as anxiety and depression [15]. SSRI has serotonin reuptake reduction in specific neurons, causing an increase in the brain serotonin and reduce the symptoms of mental health disorders. SSRI, in general, are safer than others but have their own side effects, which are usually sexual, metabolic, and gastrointestinal [16-18].

In patients with OCD, the response to medication should be evaluated after a time period of about $8-12-w$. This time is usually more than the time period of the response to medication in patients with depression (3-4 w). Of course, the time may vary slightly, but mainly in the OCD treatment, the patient needs more time and dosage as well.

The main goal of medication is to reduce obsessive thoughts and actions so that the patient can naturally reduce activity and performance. Usually, $25-35 \%$ in the Yale-Brown Obsessive Compulsive Scale (Y-BOCS) considered as a favorable clinical response [19]. Currently, about $40 \%$ to $60 \%$ of patients show significant improvement by taking first SSRI drug, but few responses to drugs are very high [20,21].

The latest available systematic reviews on antipsychotic therapies in resistant OCD are done from 2005 to 2006 [22,23]; thus, many new relevant researches have been published, an update of the current available literature seems necessary. We aimed to systematically 
evaluate the effects of SSRI compared with SGAs considering all published randomized clinical trials (RCT) studies for people with OCD.

\section{Pharmacology}

It seems that the FGAs and SGAs inhibit postsynaptic DAT D2 receptors in the brain. Several studies attest to the role of DAT D2 receptors in the antipsychotic drugs activity, including connections between these drugs and receptor and the clinical potency. Functional imaging studies show that $60-65 \%$ of DAT D2 receptors should be tackled by the effect of antipsychotic medications [24,25]. SGAs also bind to serotonin receptors that increase their affinity for connection to DAT D2 receptors, which this effect is not seen in the FGAs [26]. Largely for this reason, serotonin receptors may reduce the risk of extrapyramidal side effects of most second-generation drugs, known as atypical antipsychotics, compared to the first-generation agents, especially in case of high potency drugs [27]. SSRIs primarily inhibit serotonin transporter (SERT) and the uptake of serotonin (5-HT) in the brain. These drugs also have controversial effects on DAT and norepinephrine transporters (NET). SSRI play a role in improving depression symptoms through inhibiting the binding of the neurotransmitter, serotonin (5-HT), to SERT, which results in increased 5-HT concentration and it's binding to postsynaptic receptors.

\section{METHODS}

\section{Types of studies}

We included all double-blind, randomized controlled trials.

\section{Types of participants}

We included studies in which people with a primary diagnosis of OCD according to Diagnostic and Statistical Manual-III (DSM-III)/DSMIV or International Classification of Diseases-10 both children and adults. We did not exclude any OCD trials in participants with a serious concomitant medical illness.

\section{Types of interventions}

SGAs and SSRIs could be given as a monotherapy or as adjunctive therapy compared with placebo or other antidepressants. There were no limits in terms of study duration.

\section{Search methods for identification of studies}

We searched PubMed and Cochrane Central Register of Controlled Trials (CENTRAL) systematically up to 29/01/2016. The search terms used were: ((Obsess* or compul* or OCD) and "atypical antipsychotic*" or "second-generation antipsychotic*" or "secondgeneration antipsychotic*") and ((obsess* or compul* or OCD) and "atypical antipsychotic*" or "second-generation antipsychotic*" or "second-generation antipsychotic*" or "SSRI")). We also searched www. clinicaltrials.gov using search terms for intervention and condition, e.g., SGAs AND OCD, SSRI and OCD. No language restrictions were applied.

\section{RESULTS}

\section{SSRI in refractory $O C D$}

Through databases, we found 78 RCTs, which included SSRI compared with routine drug therapy or placebo (Table 1). Out of these 78 studies,
62 studies were conducted on adult patients with OCD, comprising 7920 cases. While only 16 RCTs were performed on children and adolescents with OCD, including 1313 people.

Comparing these two groups of patients revealed that fluvoxamine was most frequent drugs used in adults; hence, most frequent drugs used in children were sertraline (Fig. 1).

Of 78 studies, fluvoxamine was the most frequent drugs used in patients with refractory OCD with $26(33.33 \%)$ frequency followed by paroxetine, sertraline, and fluoxetine (Fig. 2).

Four SSRIs have been approved for the adult OCD treatment by the FDA so far, including fluvoxamine, fluoxetine, sertraline, and paroxetine. Among these five SSRIs, only four drugs have also been approved for treatment of pediatric OCD, including clomipramine, fluoxetine, fluvoxamine, and sertraline [102]. Fluvoxamine is one of the SSRI drugs, which is primarily used to treat OCD, social anxiety disorder, major depression, management of obesity, and bulimia, schizophrenia, and panic disorder. Many researchers believe that the imbalance in neurotransmitters causes depression and other mental disorders. Fluvoxamine inhibits serotonin reuptake that causes mania and euphoria. Furthermore, fluvoxamine has also been approved by the Food and Drug Administration (FDA) for the OCD treatment. Antidepressants such as fluvoxamine may increase the risk of suicide in children and young adults even in the first few weeks of consumption. This drug was the first SSRI licensed for use in adults, as well as for children, in OCD in the United States and Japan [103]. A number of RCT studies have confirmed the efficacy of fluvoxamine in improving the symptoms of OCD, and subsequently reducing the disruption it causes in daily life as well $[28,33,39,42,44,46]$. Trend of using SSRI in RCTs on refractory OCD also showed a decreasing pattern for fluvoxamine and paroxetine (Fig. 3).

No SSRI has been verified to be more effective than others in patients with OCD. Nevertheless, patients may individually respond more satisfactorily to one SSRI than to another. The most effective SSRI in any given patient is difficult to predict. Therefore, considering cost, available formulations, side effect profile, and half-life may help the selection. Among different SSRIs, currently only generic forms of clomipramine, citalopram, fluoxetine, fluvoxamine, and paroxetine are available.

\section{SGAs in refractory $\mathrm{OCD}$}

We searched PubMed and Cochrane Central Register of Controlled Trials (CENTRAL), which lead us to 24 clinical trial studies (Table 2). These 24 trials were conducted on adult patients with OCD, including 992 cases.

Of 24 studies, risperidone and quetiapine were the most frequent SGA drugs used in patients with refractory OCD with $8(33.33 \%)$ frequencies in both (Fig. 4).

The trend of using SGAs in RCTs on refractory OCD also showed an increasing pattern only for aripiprazole, whereas in the case of olanzapine and quetiapine was decreasing (Fig. 5).
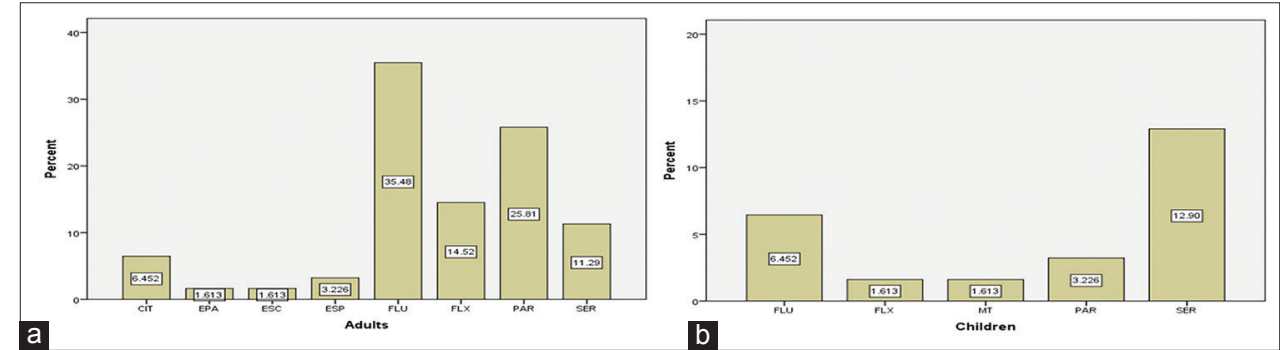

Fig. 1: Comparing the frequency of using serotonin-specific reuptake inhibitors in adult (a) versus children, (b) patients with obsessive-compulsive disorder 


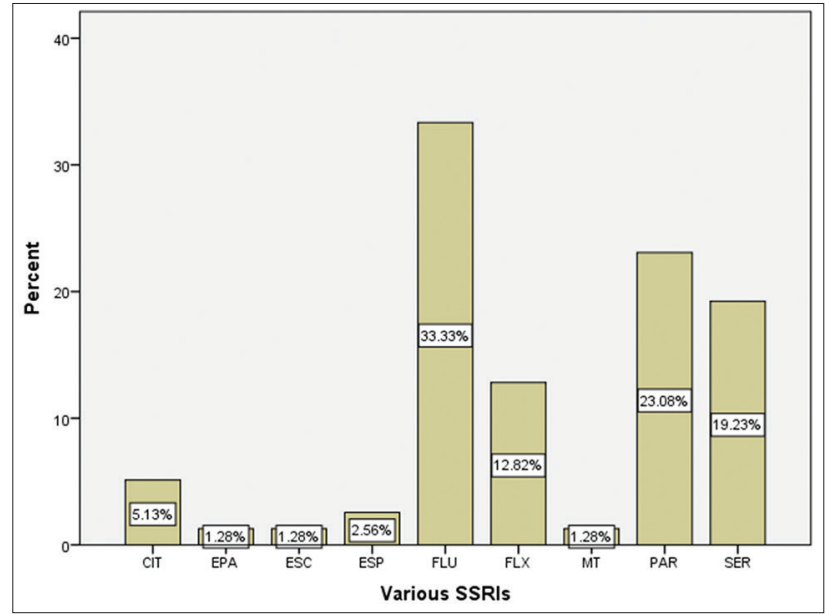

Fig. 2: Percentage of used various serotonin-specific reuptake inhibitors in clinical trials on patients with refractory obsessivecompulsive disorder. FLU: Fluvoxamine, ESC: Escitalopram, MT: Medication therapy, including citalopram (CIT), ESC, FLU, paroxetine (PAR), Clomipramine (CLO), Venlafaxine (VEN);

Risperidone (RIS); Fluoxetine (FLX); Sertraline (SER)

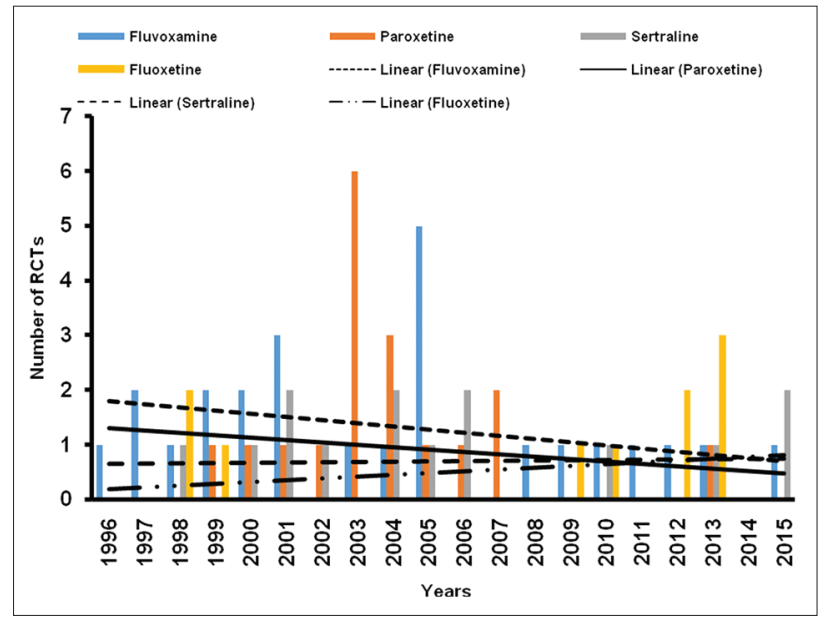

Fig. 3: Trends of the most frequent used serotonin-specific reuptake inhibitors in obsessive-compulsive disorder from 1996 to 2016

\section{Mixed SSRI and SGAs in refractory OCD}

Through our search, we included only 4 RCTs with 252 participants on SGAs plus SSRIs in refractory OCD patients (Table 3). All trials investigated the effects of adding SGAs to SSRIs with the duration of more than 6-w.

\section{DISCUSSION}

Drug treatment is one of the most common methods of treatment of patients with clinical mental health disorders such as OCD. Although using SSRI drug in trials is beneficial with a selective efficacy in OCD, up to $40 \%$ to $60 \%$ of OCD patients claim no satisfactory outcome $[20,21]$. As yet little is known about the efficacy and side effects of SGAs and SSRI in people suffering from OCD.

Due to the irrational and excessive nature of OCD, the treatment of refractory OCD is the major concern of psychiatrists. Unfortunately, despite advance in therapy and developing new and effective treatment modalities in the treatment of OCD, majority of patients suffering from OCD and at an increased risk of developing the disorder. One of the reasons can be the diverse nature of OCD. Considering DSM-III, DSM-

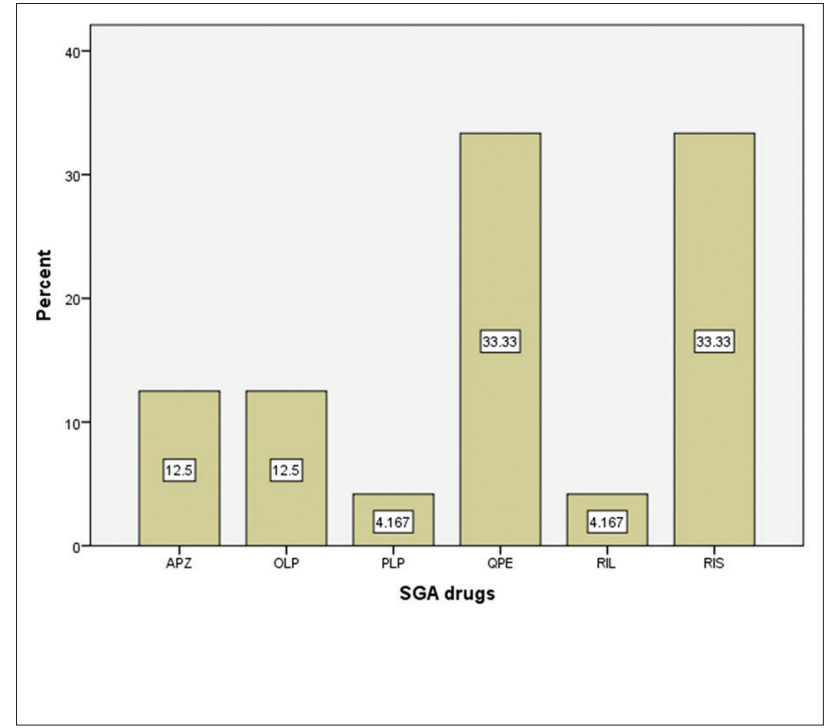

Fig. 4: Percentage of used various SGA in clinical trials on patients with refractory obsessive-compulsive disorder. PLP: Paliperidone, APZ: Aripiprazole, QPE: Quetiapine, OLP: Olanzapine, RIL: Riluzole, RIS: Risperidone

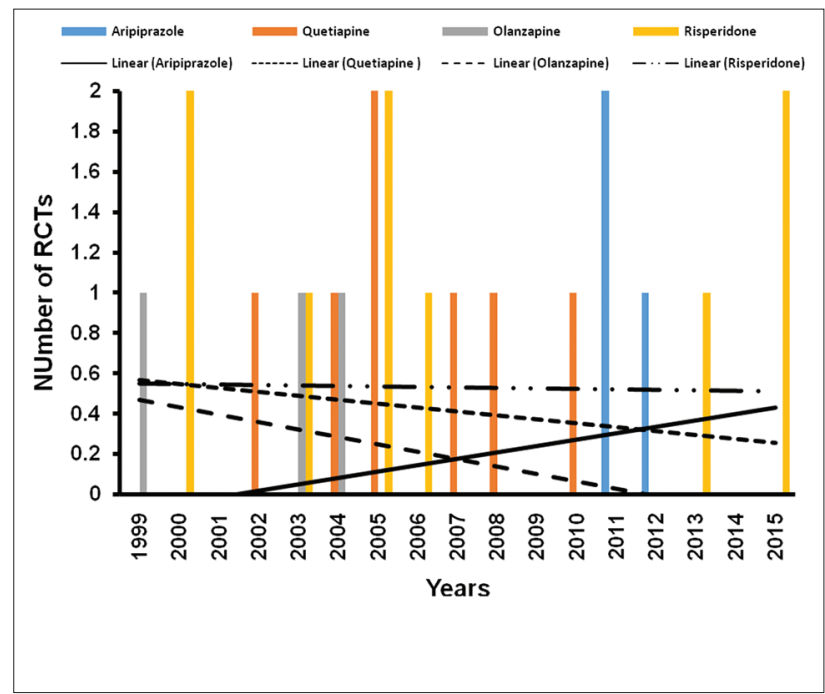

Fig. 5: Trends of the most frequent used serotonin-specific reuptake inhibitors in obsessive-compulsive disorder from 1996 to 2016

III-R, and DSM-IV, OCD was classified as an anxiety disorder, whereas in ICD-10, this disorder was separated from the anxiety disorders. Recent advances in understanding illness anxiety have led to the question of whether OCD should no longer be classified as anxiety disorders in DSM-V or not $[131,132]$.

According to the trials reviewed in this article can say that both fluvoxamine and quetiapine are the drug of choice and first-line agents in the early treatment of OCD. However, due to the growing trend of aripiprazole seems that soon this antipsychotic agent replaced the use of quetiapine in the treatment of refractory OCD. As the treatment of refractory OCD generally requires high doses of SSRIs, this higher dose increases the side effects, especially loss of sexual drive [133]. In agreement with our findings, Irons in a review studied the use of fluvoxamine in the treatment of mental health disorders, claimed that this SSRI agent is well-tolerated and does not cause sedation or 
Table 1: Summary of recent clinical trials that was found by initial search

\begin{tabular}{|c|c|c|c|c|c|c|}
\hline \multirow[t]{2}{*}{ Design } & \multirow[t]{2}{*}{ Outcome } & \multicolumn{2}{|c|}{ Study arms } & \multirow[t]{2}{*}{ Follow-up } & \multirow[t]{2}{*}{ Participant* } & \multirow{2}{*}{$\begin{array}{l}\text { Author, year, } \\
\text { country }\end{array}$} \\
\hline & & Drug & Placebo & & & \\
\hline $\begin{array}{l}\text { Randomized } \\
\text { double-blind clinical trial }\end{array}$ & $\begin{array}{l}\text { Y-BOCS } \\
\text { score, patient } \\
\text { characteristics } \\
\text { predictive of } \\
\text { assignment } \\
\text { compliance }\end{array}$ & FLU & $\mathrm{BT}$ & 12 week & $\begin{array}{l}48 \text { patients with } \mathrm{OCD} \\
\text { resistant to a BT }\end{array}$ & $\begin{array}{l}\text { Landsheer } \\
\text { et al. 2015, } \\
\text { Netherlands [28] }\end{array}$ \\
\hline $\begin{array}{l}\text { A multi-site, parallel, } \\
\text { double-blind, } \\
\text { randomized, placebo } \\
\text { controlled trial }\end{array}$ & $\begin{array}{l}\text { CGI-SA, CY-BOCS, } \\
\text { CGI-SI }\end{array}$ & SER & РBO & 8 week & 44 youths with OCD & $\begin{array}{l}\text { Bussing et al. } \\
\text { 2015, USA [29] }\end{array}$ \\
\hline $\begin{array}{l}\text { Randomized controlled } \\
\text { trial }\end{array}$ & $\begin{array}{l}\text { CY-BOCS total } \\
\text { score, clinical } \\
\text { response }\end{array}$ & SER & CBT & 16 week & $\begin{array}{l}54 \text { children and } \\
\text { adolescents (age } \\
7-17 \text { years) with primary } \\
\text { OCD }\end{array}$ & $\begin{array}{l}\text { Skarphedinsson } \\
\text { et al. 2015, } \\
\text { Norway [30] }\end{array}$ \\
\hline $\begin{array}{l}\text { Randomized, parallel } \\
\text { assignment, single blind } \\
\text { clinical trial }\end{array}$ & $\begin{array}{l}\text { CGI-SA, CY-BOCS, } \\
\text { CGI-SI }\end{array}$ & MT & $\mathrm{MT}+\mathrm{CBT}$ & 12 week & $\begin{array}{l}124 \text { youth (aged } 7 \text { to } \\
17 \text { years) with a primary } \\
\text { OCD }\end{array}$ & $\begin{array}{l}\text { Conelea et al. } \\
\text { 2014, USA [31] }\end{array}$ \\
\hline Double-blind clinical trial & DUOCS & FLX & $\mathrm{FLX}+\mathrm{CBT}$ & 13 week & 30 cases with OCD & $\begin{array}{l}\text { Giasuddin } \\
\text { et al. 2013, } \\
\text { Bangladesh [32] }\end{array}$ \\
\hline $\begin{array}{l}\text { randomized double-blind } \\
\text { placebo-controlled }\end{array}$ & $\begin{array}{l}\text { Y-BOCS score, } \\
\text { efficacy, } \\
\text { tolerability }\end{array}$ & FLU & РBO & 8 week & 42 patients with OCD & $\begin{array}{l}\text { Ghaleiha et al. } \\
\text { 2013, Iran [33] }\end{array}$ \\
\hline $\begin{array}{l}\text { Double-blind } \\
\text { placebo-controlled } \\
\text { randomized clinical trial }\end{array}$ & YBOCS & PAR & РBO & 12 week & 36 adults with OCD & $\begin{array}{l}\text { Humble } \\
\text { et al. 2013, } \\
\text { Sweden [34] }\end{array}$ \\
\hline Randomized open trial & YBOCS & FLX & CBT & 12 week & 160 cases with OCD & $\begin{array}{l}\text { Jakubovski } \\
\text { et al. 2013, } \\
\text { Germany [35] }\end{array}$ \\
\hline $\begin{array}{l}\text { Double-blind randomized } \\
\text { controlled trial }\end{array}$ & TEASAP score & SER & РBO & 4 week & $\begin{array}{l}56 \text { youth (aged } 7-17 \text { ) } \\
\text { with OCD }\end{array}$ & $\begin{array}{l}\text { Bussing et al. } \\
\text { 2013, USA [36] }\end{array}$ \\
\hline Randomized clinical trial & YBOCS & FLX & СВТ & 12 week & $\begin{array}{l}29 \text { adult patients with } \\
\text { OCD }\end{array}$ & $\begin{array}{l}\text { Hoexter et al. } \\
\text { 2013, Brazil [37] }\end{array}$ \\
\hline $\begin{array}{l}\text { Randomized } \\
\text { placebo-controlled } \\
\text { clinical trial }\end{array}$ & YBOCS & FLX & РBO & 8 week & $\begin{array}{l}31 \text { adult patients with } \\
\text { OCD }\end{array}$ & $\begin{array}{l}\text { Sayyah et al. } \\
\text { 2012, Iran [38] }\end{array}$ \\
\hline $\begin{array}{l}\text { Randomized controlled } \\
\text { trial }\end{array}$ & YBOCS & FLU & СBT & 12 week & 118 subjects with OCD & $\begin{array}{l}\text { van Balkom } \\
\text { et al. 2012, } \\
\text { Netherlands [39] }\end{array}$ \\
\hline $\begin{array}{l}\text { Randomized, } \\
\text { double-blinded } \\
\text { controlled clinical trial }\end{array}$ & YBOCS & FLX & CBT & 12 week & $\begin{array}{l}38 \text { adult patients with } \\
\text { OCD }\end{array}$ & $\begin{array}{l}\text { Hoexter et al. } \\
\text { 2012, Brazil [40] }\end{array}$ \\
\hline $\begin{array}{l}\text { Randomized, } \\
\text { single-blinded clinical } \\
\text { trial }\end{array}$ & $\begin{array}{l}\text { Y-BOCS score, } \\
\text { obsessions }\end{array}$ & SER & CBT & 12 week & $\begin{array}{l}46 \text { patients with a } \\
\text { primary OCD }\end{array}$ & $\begin{array}{l}\text { Borges et al. } \\
\text { 2011, Brazil [41] }\end{array}$ \\
\hline $\begin{array}{l}\text { randomized double-blind } \\
\text { placebo-controlled }\end{array}$ & $\begin{array}{l}\text { Y-BOCS score, } \\
\text { symptoms of } \\
\text { obsessions and } \\
\text { compulsions }\end{array}$ & $\mathrm{CEL}+\mathrm{FLU}$ & PBO+FLU & 8 week & 50 patients with $\mathrm{OCD}$ & $\begin{array}{l}\text { Sayyah et al. } \\
\text { 2011, Iran [42] }\end{array}$ \\
\hline $\begin{array}{l}\text { Randomized, } \\
\text { placebo-controlled trial }\end{array}$ & YBOCS-SC & SER & РВО & 12 week & $\begin{array}{l}112 \text { youth (aged 7-17) } \\
\text { with OCD }\end{array}$ & $\begin{array}{l}\text { Garcia et al. } \\
\text { 2010, USA [43] }\end{array}$ \\
\hline $\begin{array}{l}\text { randomized double-blind } \\
\text { placebo-controlled }\end{array}$ & $\begin{array}{l}\text { Y-BOCS score, side } \\
\text { effects }\end{array}$ & FLU & S.M & 8 week & 35 patients with OCD & $\begin{array}{l}\text { Sayyah et al. } \\
\text { 2010, Iran [44] }\end{array}$ \\
\hline $\begin{array}{l}\text { Double-blind randomized } \\
\text { clinical trial }\end{array}$ & YBOCS & FLX & PBO & 8 week & $\begin{array}{l}42 \text { adult patients with } \\
\text { OCD }\end{array}$ & $\begin{array}{l}\text { Soltani et al. } \\
\text { 2010, Iran [45] }\end{array}$ \\
\hline $\begin{array}{l}\text { Randomized, controlled } \\
\text { trials }\end{array}$ & YBOCS & FLU & PAR & 12 week & 44 adults with OCD & $\begin{array}{l}\text { Matsunaga et al. } \\
\text { 2009, Japan [46] }\end{array}$ \\
\hline $\begin{array}{l}\text { Randomized, double } \\
\text { blind, fixed-does }\end{array}$ & CY-BOCS, CGI & FLX & CIT & 6 week & $\begin{array}{l}29 \text { children and } \\
\text { adolescents ( } 7-18 \text { years) } \\
\text { with OCD }\end{array}$ & $\begin{array}{l}\text { Alaghband-Rad } \\
\text { et al. 2009, } \\
\text { Canada [47] }\end{array}$ \\
\hline $\begin{array}{l}\text { Randomized } \\
\text { double-blind } \\
\text { placebo-controlled }\end{array}$ & $\begin{array}{l}\text { Y-BOCS score, } \\
\text { efficacy, } \\
\text { tolerability }\end{array}$ & ESC & PBO & 12 week & 466 adults with OCD & $\begin{array}{l}\text { Stein et al. 2008, } \\
\text { South Africa [48] }\end{array}$ \\
\hline Randomized to open label & YBOCS, CGI-I & $\mathrm{FLU}+\mathrm{GBP}$ & FLU & 8 week & 40 patients with IC-IUD & $\begin{array}{l}\text { Onder et al. 2008, } \\
\text { Turkey [49] }\end{array}$ \\
\hline
\end{tabular}


Table 1: (Continued)

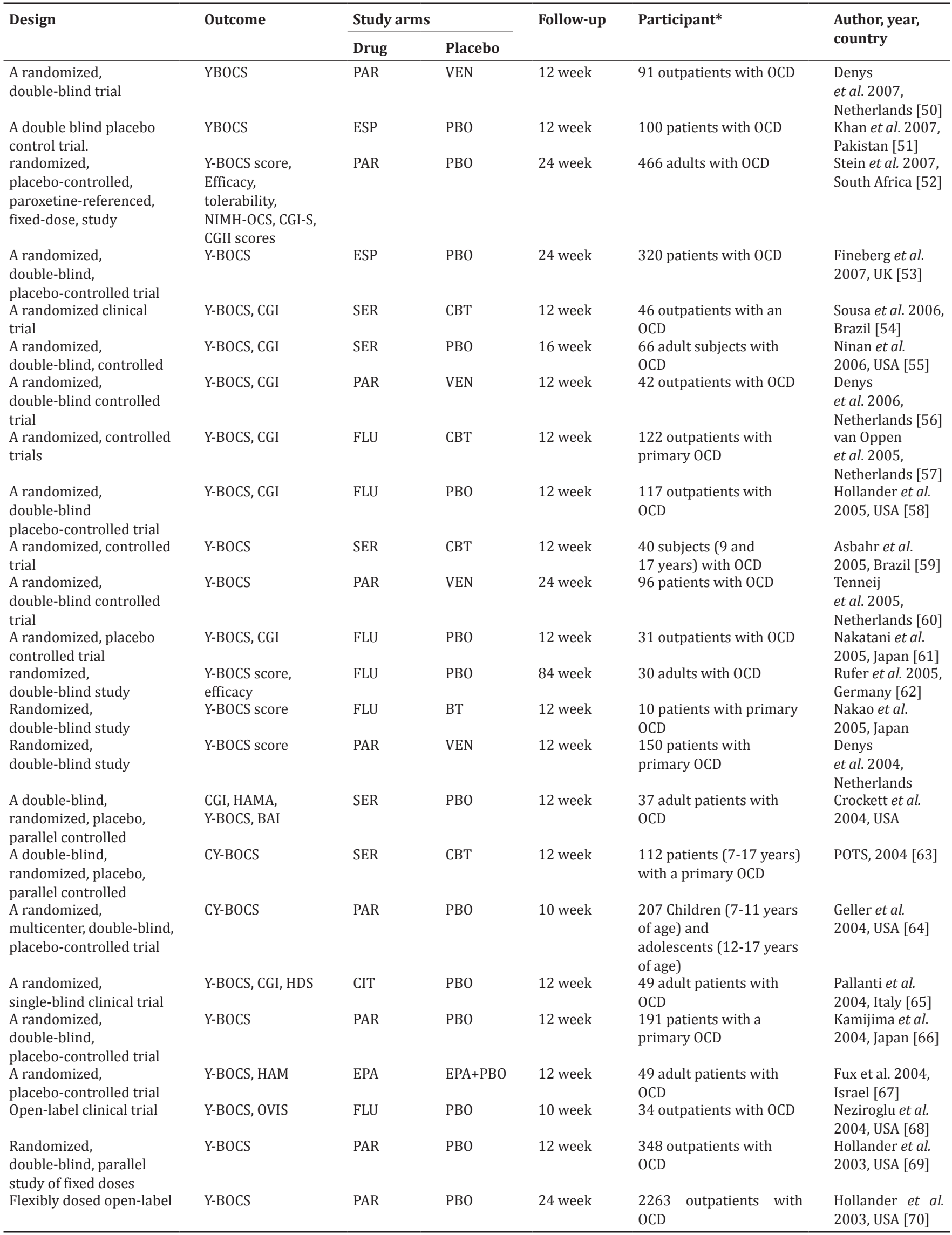


Table 1: (Continued)

\begin{tabular}{|c|c|c|c|c|c|c|}
\hline \multirow[t]{2}{*}{ Design } & \multirow[t]{2}{*}{ Outcome } & \multicolumn{2}{|c|}{ Study arms } & \multirow[t]{2}{*}{ Follow-up } & \multirow[t]{2}{*}{ Participant* } & \multirow{2}{*}{$\begin{array}{l}\text { Author, year, } \\
\text { country }\end{array}$} \\
\hline & & Drug & Placebo & & & \\
\hline $\begin{array}{l}\text { Double-blind, fixed-dose, } \\
\text { parallel trial }\end{array}$ & $\begin{array}{l}\text { long-term efficacy, } \\
\text { safety, and } \\
\text { impact on relapse } \\
\text { prevention }\end{array}$ & PAR & PBO & 24 week & $\begin{array}{l}3105 \text { outpatients with } \\
\text { OCD }\end{array}$ & $\begin{array}{l}\text { Hollander et al. } \\
\text { 2003, USA [71] }\end{array}$ \\
\hline $\begin{array}{l}\text { A randomized, } \\
\text { double-blind, } \\
\text { placebo-controlled trial }\end{array}$ & Y-BOCS, HAM & PAR & VEN & 12 week & $\begin{array}{l}150 \text { patients with } \\
\text { primary OCD }\end{array}$ & $\begin{array}{l}\text { Denys } \\
\text { et al. 2003, } \\
\text { Netherlands [72] }\end{array}$ \\
\hline $\begin{array}{l}\text { Randomized, } \\
\text { double-blind study }\end{array}$ & $\begin{array}{l}\text { Y-BOCS score, } \\
\text { efficacy }\end{array}$ & PAR & VEN & 12 week & $\begin{array}{l}140 \text { patients with } \\
\text { primary OCD }\end{array}$ & $\begin{array}{l}\text { Denys } \\
\text { et al. 2003, } \\
\text { Netherlands [73] }\end{array}$ \\
\hline $\begin{array}{l}\text { A randomized, } \\
\text { double-blind, } \\
\text { placebo-controlled trial }\end{array}$ & Y-BOCS, CGI & PAR & PBO & 16 week & 193 adult OCD patients & $\begin{array}{l}\text { Geller et al. } \\
\text { 2003, USA [74] }\end{array}$ \\
\hline $\begin{array}{l}\text { A randomized, } \\
\text { double-blind, } \\
\text { placebo-controlled trial }\end{array}$ & Y-BOCS, CGI & FLU & PBO & 12 week & 253 adult OCD patients & $\begin{array}{l}\text { Hollander et al. } \\
\text { 2003, USA [75] }\end{array}$ \\
\hline $\begin{array}{l}\text { A randomized, } \\
\text { double-blind, } \\
\text { placebo-controlled trial }\end{array}$ & CY-BOCS, CGI & FLU & PBO & 8 week & 43 young OCD patients & $\begin{array}{l}\text { Liebowitz et al. } \\
\text { 2002, USA [76] }\end{array}$ \\
\hline $\begin{array}{l}\text { A randomized, } \\
\text { single-blind, controlled } \\
\text { trial }\end{array}$ & CY-BOCS, CGI & PAR & VEN & 12 week & $73^{\circ} \mathrm{CD}$ patients & $\begin{array}{l}\text { Albert et al. } \\
\text { 2002, Italy [77] }\end{array}$ \\
\hline Open-label trial & Y-BOCS & CIT & PBO & 12 week & $39^{\circ} \mathrm{CD}$ patients & $\begin{array}{l}\text { Pallanti et al. } \\
\text { 2002, Italy [21] }\end{array}$ \\
\hline $\begin{array}{l}\text { Randomized, } \\
\text { single-blind, placebo } \\
\text { controlled study }\end{array}$ & $\begin{array}{l}\text { Y-BOCS score, } \\
\text { efficacy }\end{array}$ & FLU & PBO & 52 week & $\begin{array}{l}130 \text { patients with } \\
\text { primary OCD** }\end{array}$ & $\begin{array}{l}\text { Romano et al. } \\
\text { 2001, USA [78] }\end{array}$ \\
\hline $\begin{array}{l}\text { A randomized, } \\
\text { double-blind, controlled } \\
\text { trial }\end{array}$ & YBOCS, CGI & SER & FLU & 24 week & 150 patients were OCD & $\begin{array}{l}\text { Bergeron } \\
\text { et al. 2002, } \\
\text { Canada [79] }\end{array}$ \\
\hline $\begin{array}{l}\text { A large randomized } \\
\text { placebo-controlled trial }\end{array}$ & YBOCS, CGI & CIT & PBO & 12 week & 71 patients were OCD & $\begin{array}{l}\text { Stein et al. 2001, } \\
\text { South Africa [80] }\end{array}$ \\
\hline $\begin{array}{l}\text { A double-blind, } \\
\text { placebo-controlled }\end{array}$ & $\begin{array}{l}\text { CY-BOCS, CGI-S, } \\
\text { CGI-I }\end{array}$ & SER & PBO & 12 week & $\begin{array}{l}132 \text { Children ( } 6-12 \text { years; } \\
n=72) \text { and } \\
\text { adolescents (13-18 years; } \\
n=65 \text { ) with OCD }\end{array}$ & $\begin{array}{l}\text { Cook et al. 2001, } \\
\text { USA [82] }\end{array}$ \\
\hline $\begin{array}{l}\text { A randomized, } \\
\text { double-blind, controlled } \\
\text { trial }\end{array}$ & CY-BOCS & FLU & PBO & 13 week & $\begin{array}{l}103 \text { Children ( } 7-17 \text { years) } \\
\text { with OCD }\end{array}$ & $\begin{array}{l}\text { Geller et al. } \\
\text { 2001, USA [83] }\end{array}$ \\
\hline $\begin{array}{l}\text { A randomized, } \\
\text { double-blind trial }\end{array}$ & YBOCS, BABS & PAR & PBO & 12 week & 36 patients were OCD & $\begin{array}{l}\text { Humble } \\
\text { et al. } 2001, \\
\text { Sweden [84] }\end{array}$ \\
\hline $\begin{array}{l}\text { A randomized, } \\
\text { double-blind, } \\
\text { placebo-controlled, } \\
\text { multicenter study }\end{array}$ & CY-BOCS & FLU & PBO & 10 week & $\begin{array}{l}120 \text { Children ( } 7-17 \text { years) } \\
\text { with OCD }\end{array}$ & $\begin{array}{l}\text { Riddle et al. } \\
\text { 2001, USA [85] }\end{array}$ \\
\hline $\begin{array}{l}\text { Double-blind, } \\
\text { placebo-controlled study }\end{array}$ & Y-BOCS & FLU & PBO & 10 week & 33 patients with OCD & $\begin{array}{l}\text { Peter et al. 2000, } \\
\text { Germany [86] }\end{array}$ \\
\hline $\begin{array}{l}\text { A double-blind, } \\
\text { placebo-controlled trial }\end{array}$ & $\begin{array}{l}\text { Y-BOCS, HAM-A, } \\
\text { MADRS }\end{array}$ & PAR & PBO & 6 week & $\begin{array}{l}14 \text { treatment-resistant } \\
\text { OCD patients }\end{array}$ & $\begin{array}{l}\text { Dannon et al. } \\
\text { 2000, Israel [87] }\end{array}$ \\
\hline $\begin{array}{l}\text { Randomized, } \\
\text { double-blind study }\end{array}$ & $\begin{array}{l}\text { Children's Y-BOCS } \\
\text { score, NIMH-OCS, } \\
\text { CGI-S, CGII scores }\end{array}$ & FLU & BT & 12 week & $\begin{array}{l}10 \text { children/adolescents } \\
\text { with OCD }\end{array}$ & $\begin{array}{l}\text { Neziroglu et al. } \\
\text { 2000, USA [88] }\end{array}$ \\
\hline $\begin{array}{l}\text { A randomized, } \\
\text { double-blind, } \\
\text { placebo-controlled, } \\
\text { multicenter study }\end{array}$ & $\begin{array}{l}\text { Y-BOCS, HAM-A, } \\
\text { MADRS }\end{array}$ & SER & DPM & 12 week & 166 patients with OCD & $\begin{array}{l}\text { Hoehn-Saric } \\
\text { et al. } 2000, \\
\text { USA [89] }\end{array}$ \\
\hline $\begin{array}{l}\text { Randomized, open-label } \\
\text { trial }\end{array}$ & Y-BOCS & CIT & $\mathrm{CIT}+\mathrm{CLO}$ & 12 week & $\begin{array}{l}\text { Sixteen adult outpatients } \\
\text { with OCD }\end{array}$ & $\begin{array}{l}\text { Pallanti et al. } \\
\text { 1999, Italy [90] }\end{array}$ \\
\hline Open-label trial & $\begin{array}{l}\text { CY-BOCS, HAM, } \\
\text { CGI }\end{array}$ & PAR & PBO & 12 week & $\begin{array}{l}20^{\circ} \mathrm{CD} \text { outpatients ( } 8 \text { to } \\
17 \text { years) }\end{array}$ & $\begin{array}{l}\text { Rosenberg et al. } \\
\text { 1999, USA [91] }\end{array}$ \\
\hline $\begin{array}{l}\text { Double-blind } \\
\text { placebo-controlled trial }\end{array}$ & YBOCS, CGI & FLX & PBO & 8 week & 53 patients with OCD & $\begin{array}{l}\text { Zitterl et al.1999, } \\
\text { Austria [92] }\end{array}$ \\
\hline
\end{tabular}


Table 1: (Continued)

\begin{tabular}{|c|c|c|c|c|c|c|}
\hline \multirow[t]{2}{*}{ Design } & \multirow[t]{2}{*}{ Outcome } & \multicolumn{2}{|c|}{ Study arms } & \multirow[t]{2}{*}{ Follow-up } & \multirow[t]{2}{*}{ Participant* } & \multirow{2}{*}{$\begin{array}{l}\text { Author, year, } \\
\text { country }\end{array}$} \\
\hline & & Drug & Placebo & & & \\
\hline \multirow{2}{*}{$\begin{array}{l}\text { Double-blind } \\
\text { placebo-controlled trial } \\
\text { Randomized, } \\
\text { placebo-controlled study }\end{array}$} & YBOCS, CGI & FLU & PBO & 10 week & $50^{\circ} \mathrm{C}$ patients & $\begin{array}{l}\text { Mundo et al. } \\
\text { 1999, Italy [93] }\end{array}$ \\
\hline & $\begin{array}{l}\text { Anxiety } \\
\text { Discomfort Scale, } \\
\text { Y-BOCS score, } \\
\text { and the Padua } \\
\text { Inventory-Revised }\end{array}$ & FLU & CT & 16 week & $\begin{array}{l}117 \text { patients with } \\
\text { primary OCD }\end{array}$ & $\begin{array}{l}\text { van Balkom } \\
\text { et al. 1998, } \\
\text { Netherlands [94] }\end{array}$ \\
\hline $\begin{array}{l}\text { Double-blind } \\
\text { placebo-controlled trial }\end{array}$ & YBOCS, CGI & FLX & PBO & 12 week & $14^{\circ} \mathrm{C}$ patients & $\begin{array}{l}\text { Greenberg et al. } \\
\text { 1998, USA [95] }\end{array}$ \\
\hline $\begin{array}{l}\text { Randomized, } \\
\text { double-blind, } \\
\text { placebo-controlled trial }\end{array}$ & $\begin{array}{l}\text { CY-BOCS, NIMH } \\
\text { GOCS, CGI }\end{array}$ & SER & PBO & 12 week & $\begin{array}{l}107 \text { children ( } 6 \text { to } \\
12 \text { years) and } 80 \\
\text { adolescents ( } 13 \text { to } \\
17 \text { years) with OCD }\end{array}$ & $\begin{array}{l}\text { March et al. } \\
\text { 1998, USA [96] }\end{array}$ \\
\hline $\begin{array}{l}\text { Randomized, } \\
\text { double-blind, } \\
\text { placebo-controlled trial }\end{array}$ & YBOCS & FLU & PBO & 8 week & $60^{\circ} \mathrm{C}$ patients & $\begin{array}{l}\text { Hohagen } \\
\text { et al. } 1998, \\
\text { Germany [97] }\end{array}$ \\
\hline $\begin{array}{l}\text { Multicenter, } \\
\text { placebo-controlled, } \\
\text { fixed-dose trial }\end{array}$ & YBOCS & FLX & PBO & 10 week & $\begin{array}{l}35 \text { patients with primary } \\
\text { OCD }\end{array}$ & $\begin{array}{l}\text { Ackerman et al. } \\
\text { 1998, USA [98] }\end{array}$ \\
\hline $\begin{array}{l}\text { Randomized, } \\
\text { single-blind, placebo } \\
\text { controlled study }\end{array}$ & $\begin{array}{l}\text { Y-BOCS, NIMH-OC } \\
\text { scale, the CGIIS } \\
\text { Scale, and the HRS } \\
\text { for depression }\end{array}$ & FLU & CIT & 10 week & $\begin{array}{l}30 \text { patients with primary } \\
\text { OCD }\end{array}$ & $\begin{array}{l}\text { Mundo et al. } \\
\text { 1997, Italy [99] }\end{array}$ \\
\hline $\begin{array}{l}\text { Double-blind controlled } \\
\text { trial }\end{array}$ & $\begin{array}{l}\text { Y-BOCS and CGIIS } \\
\text { scales }\end{array}$ & FLU & CLO & 8 week & 26 individuals with OCD & $\begin{array}{l}\text { Milanfranchi } \\
\text { et al. } 1997, \\
\text { Italy [100] }\end{array}$ \\
\hline $\begin{array}{l}\text { Double-blind controlled } \\
\text { trial }\end{array}$ & $\begin{array}{l}\text { Y-BOCS and CGIIS } \\
\text { scales }\end{array}$ & FLU & CLO & 8 week & 55 individuals with OCD & $\begin{array}{l}\text { López-Ibor } \\
\text { et al. } 1996, \\
\text { Spain [101] }\end{array}$ \\
\hline $\begin{array}{l}\text { *The diagnosis of OCD based or } \\
\text { obsessive compulsive scale, ME } \\
\text { VEN: Venlafaxine, ESC: Escitalo } \\
\text { GA: Global assessment, ADHDR } \\
\text { VEN: Venlafaxine, HDS: Hamilto } \\
\text { HRS: Hamilton rating scale, CLC }\end{array}$ & $\begin{array}{l}\text { M-IV-TR and a Y-BOCS } \\
\text { Iemantine, FLX: Fluox } \\
\text { n, SER: Sertraline, BUP } \\
\text { ention-deficit/hypera } \\
\text { pression scale, DS: De } \\
\text { omipramine, BT: Beha }\end{array}$ & $\begin{array}{l}\text { of } \geq 21 \\
\text { CIT, In } \\
\text { ropion } \\
\text { y disor } \\
\text { sive syr }\end{array}$ & $\begin{array}{l}\text { nbling se } \\
\text { SD: She } \\
\text { GI: Clini }\end{array}$ & $\begin{array}{l}\text { ased on DS } \\
\text { Escitalopran } \\
\text { ssessmen, } \\
\text { sability, rTN } \\
\text { al improven }\end{array}$ & $\begin{array}{l}\text { R and a Y-BOCS score of } \leq 19 \text {. } \\
\text { : Fluvoxamine, PAR: Paroxetin } \\
\text { Clinical global impression im } \\
\text { anscranial magnetic stimulatic } \\
\text { IN: Pindolol, CT: Cognitive th }\end{array}$ & $\begin{array}{l}\text { CS: Yale-brown } \\
\text { O: Clomipramine, } \\
\text { ement and severity, } \\
\text { IS: Risperidone, } \\
\text { y, CIT: Citalopram, }\end{array}$ \\
\hline
\end{tabular}

Table 2: Summary of recent clinical trials that was found by initial

\begin{tabular}{|c|c|c|c|c|c|c|}
\hline \multirow[t]{2}{*}{ Design } & \multirow[t]{2}{*}{ Outcome } & \multicolumn{2}{|c|}{ Study arms } & \multirow[t]{2}{*}{ Follow-up } & \multirow[t]{2}{*}{ Participant* } & \multirow{2}{*}{$\begin{array}{l}\text { Author, year, } \\
\text { country }\end{array}$} \\
\hline & & Drug & Placebo & & & \\
\hline $\begin{array}{l}\text { A pilot randomized, } \\
\text { placebo-controlled trial }\end{array}$ & Y-BOCS score obsessions & RIL & PBO & 12 week & 38 patients with OCD & $\begin{array}{l}\text { Pittenger et al. } \\
\text { 2015, USA [104] }\end{array}$ \\
\hline $\begin{array}{l}\text { A randomized, } \\
\text { placebo-controlled trial }\end{array}$ & $\begin{array}{l}\text { Y-BOCS social } \\
\text { adjustment scale-SR, } \\
\text { quality of life, HDS, BABS }\end{array}$ & RIS & PBO & 8 week & $\begin{array}{l}100 \text { patients with at least } \\
\text { moderate OCD severity }\end{array}$ & $\begin{array}{l}\text { Foa et al. } 2015, \\
\text { USA [105] }\end{array}$ \\
\hline $\begin{array}{l}\text { Double-blind, } \\
\text { placebo-controlled, pilot } \\
\text { trial }\end{array}$ & YBOCS, CGIIS & PLP & PBO & 8 week & $\begin{array}{l}34 \text { patients (aged } \\
24-67 \text { years) with OCD }\end{array}$ & $\begin{array}{l}\text { Storch et al. 2013, } \\
\text { USA [107] }\end{array}$ \\
\hline $\begin{array}{l}\text { Double-blind, } \\
\text { randomized, placebo } \\
\text { clinical trial }\end{array}$ & YBOCS & $\mathrm{APZ}$ & PBO & 12 week & 39 adult patients with OCD & $\begin{array}{l}\text { Sayyah et al. 2012, } \\
\text { Iran [108] }\end{array}$ \\
\hline $\begin{array}{l}\text { Double-blind, } \\
\text { randomized, } \\
\text { placebo-controlled trial }\end{array}$ & YBOCS & APZ & PBO & 16 week & $\begin{array}{l}201 \text { patients }(20-70 \text { years }) \\
\text { with OCD }\end{array}$ & $\begin{array}{l}\text { Muscatello et al. } \\
\text { 2011, Italy [109] }\end{array}$ \\
\hline $\begin{array}{l}\text { Randomized, } \\
\text { single-blinded clinical } \\
\text { trial }\end{array}$ & YBOCS & APZ & RIS & 12 week & $\begin{array}{l}90 \text { patients (18-65 years) } \\
\text { with OCD }\end{array}$ & $\begin{array}{l}\text { Selvi et al. 2011, } \\
\text { Turkey [110] }\end{array}$ \\
\hline $\begin{array}{l}\text { Randomized, open-label } \\
\text { trials }\end{array}$ & YBOCS, CGI & QPE & CLO & 12 week & 21 adults with OCD & $\begin{array}{l}\text { Diniz et al. 2010, } \\
\text { Brazil [111] }\end{array}$ \\
\hline $\begin{array}{l}\text { Randomized, } \\
\text { double-blind, } \\
\text { placebo-controlled trial }\end{array}$ & YBOCS & QPE & PBO & 12 week & $\begin{array}{l}40 \text { patients with primary } \\
\text { OCD }\end{array}$ & $\begin{array}{l}\text { Kordon et al. 2008, } \\
\text { Germany [112] }\end{array}$ \\
\hline
\end{tabular}


Table 2: (Continued)

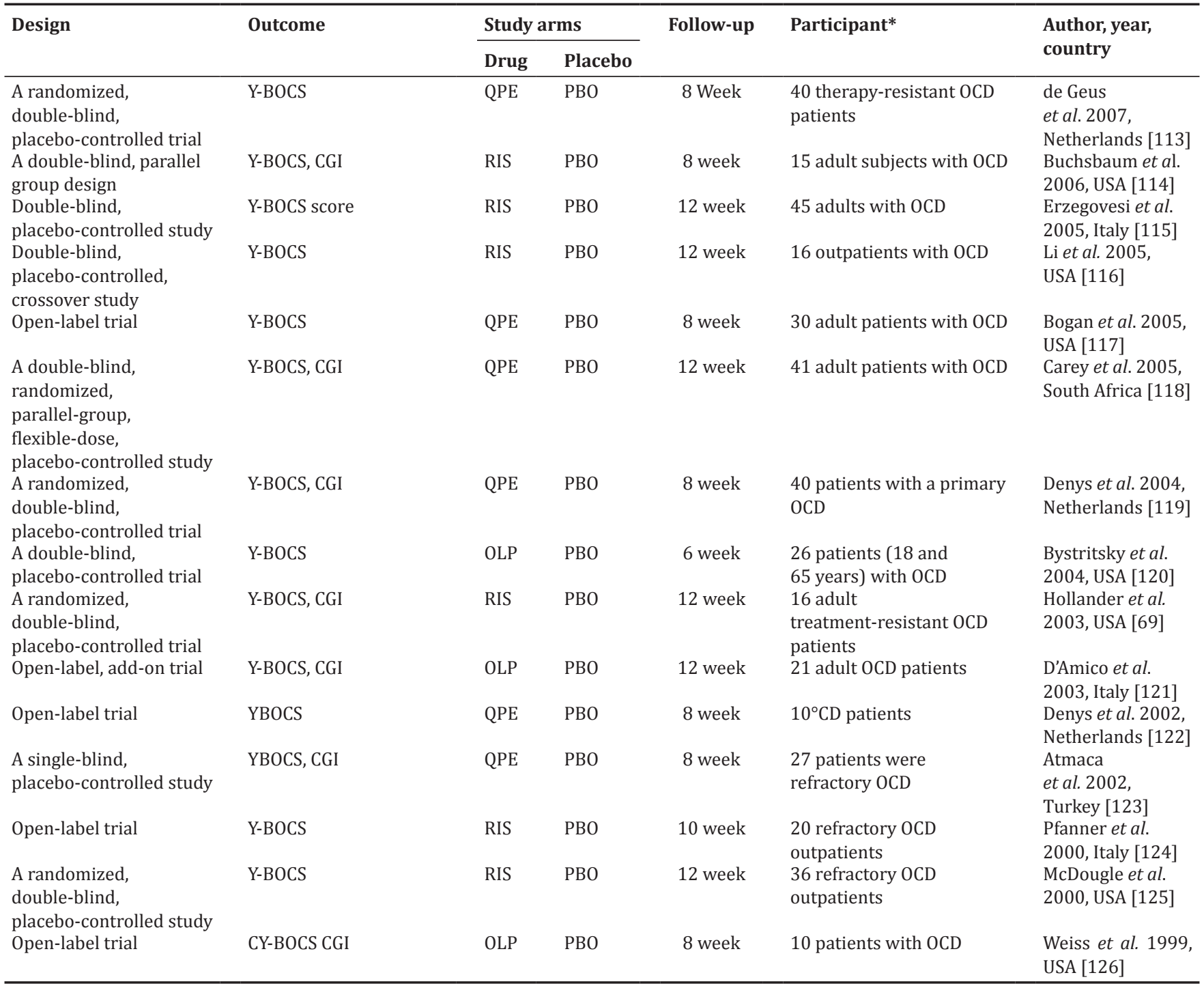

*The diagnosis of OCD based on DSM-IV-TR and a Y-BOCS score of $\geq 21$, All patients were treated with 1 of the 2 following selective serotonin reuptake inhibitors: Fluvoxamine or sertraline. Y-BOCS: Yale-brown obsessive compulsive scale, RIL: Riluzole, PBO: Placebo, RIS: Risperidone, BABS: Brown assessment of beliefs, CGI: Clinical global improvement, PLP: Paliperidone, CGIIS: Clinical global impression improvement and severity, SMTC: Stress management training condition, APZ: Aripiprazole, QPE: Quetiapine, HDS: Hamilton depression scale, OLP: Olanzapine

Table 3: Summary of recent clinical trials that was found by initial search

\begin{tabular}{|c|c|c|c|c|c|c|}
\hline \multirow[t]{2}{*}{ Design } & \multirow[t]{2}{*}{ Outcome } & \multicolumn{2}{|c|}{ Study arms } & \multirow[t]{2}{*}{ Follow-up } & \multirow[t]{2}{*}{ Participant* } & \multirow{2}{*}{$\begin{array}{l}\text { Author, year, } \\
\text { country }\end{array}$} \\
\hline & & Drug & Placebo & & & \\
\hline $\begin{array}{l}\text { Randomized, placebo-controlled, } \\
\text { clinical trial }\end{array}$ & YBOCS & $\mathrm{CIT}+\mathrm{QPE}$ & PBO & 10 week & $\begin{array}{l}46 \text { adult patients } \\
\text { with OCD }\end{array}$ & $\begin{array}{l}\text { Vulink et al. 2012, } \\
\text { Netherlands [127] }\end{array}$ \\
\hline $\begin{array}{l}\text { Randomized, double-blinded } \\
\text { controlled clinical trial }\end{array}$ & YBOCS & $\begin{array}{l}\text { QPE+FLX, } \\
\text { CIT+FLX }\end{array}$ & $\mathrm{PBO}+\mathrm{FLX}$ & 12 week & $\begin{array}{l}54 \text { patients with a } \\
\text { primary OCD }\end{array}$ & $\begin{array}{l}\text { Diniz et al. 2011, } \\
\text { Brazil [128] }\end{array}$ \\
\hline Randomized, controlled trial & YBOCS & $\mathrm{EX} / \mathrm{RP}$ & SMT & 12 week & $\begin{array}{l}108 \text { patients with } \\
\text { OCD }\end{array}$ & $\begin{array}{l}\text { Simpson et al. } \\
\text { 2008, USA [129] }\end{array}$ \\
\hline Adouble-blind,placebo-controlled & YBOCS & $\mathrm{OLP}+\mathrm{FLU}$ & $\mathrm{PBO}+\mathrm{FLU}$ & 6 week & 44 adults with OCD & $\begin{array}{l}\text { Shapira et al. 2004, } \\
\text { USA [130] }\end{array}$ \\
\hline
\end{tabular}

*The diagnosis of OCD based on DSM-IV-TR and a Y-BOCS score of $\geq 21$. Y-BOCS: Yale-brown obsessive compulsive scale, FLU: Fluvoxamine, CIT: Citalopram, QPE: Quetiapine, FLX: Fluoxetine, SMT: Stress management training, PBO: Placebo, EX/RP: Exposure/ritual prevention therapy

cognitive impairment. He also reported that this drug is associated with a low risk of sexual dysfunction, suicidality, and withdrawal reactions; thus, it is a safe SSRI agent even in overdose and has no considerable impact on cardiovascular system and body weight [103]. In a systematic review, Bloch et al. evaluated the efficacy of antipsychotic agents in treatment-refractory OCD on nine studies involving 278 participants. They claimed that there is sufficient evidence in the literature about the efficacy of haloperidol and risperidone, whereas evidence of the efficacy of quetiapine and olanzapine is unconvincing [22]. Contrary to their clime, we showed there sufficient evidence in the literature on 
risperidone, as well as quetiapine and olanzapine. The difference is may be due to the difference in the date limitation in the search strategy. Recently, Veale et al. in a systematic review and meta-analysis on 14 RCTs including risperidone, quetiapine, olanzapine, and aripiprazole [134]. They concluded that a low dose of risperidone and aripiprazole can use cautiously as an antipsychotic agent in nonresponders to SSRIs. In other reviews, Arumugham and Reddy reported that antipsychotic agents, especially risperidone and aripiprazole have shown the best evidence in refractory patients with OCD [135].

\section{CONCLUSION AND PERSPECTIVES}

As our trends show, fluvoxamine was the most frequent SSRI used in patients with refractory OCD followed by paroxetine, sertraline, and fluoxetine. Hence, risperidone and quetiapine were the most frequent SGA drugs used in patients with refractory OCD. According to the summary of our review, it seems that the first choice of early treatment programs of refractory OCD will be fluvoxamine in combination with quetiapine or aripiprazole. Recently, the treatment of patients with OCD has improved dramatically. OCD is extremely disabling and associated with considerable depression and other serious psychiatric illnesses. Therefore, this disease represents an important area of medical need. The well-known disadvantages of the traditional antipsychotics have resulted in becoming the SSRIs first-line treatment for many mental health disorders such as OCD. As our data showed among the SSRIs, fluvoxamine has been particularly well studied and used in RCTs in both children and adolescents with OCD. According to the summary of our review, it will be better when therapists use SGAs in the early treatment programs of refractory OCD. Thus, considering our reviewed, it seems that the first choice of early treatment programs of refractory OCD is fluvoxamine in combination with quetiapine or aripiprazole.

\section{REFERENCES}

1. Ruscio AM, Stein DJ, Chiu WT, Kessler RC. The epidemiology of obsessive-compulsive disorder in the National Comorbidity Survey Replication. Mol Psychiatry 2010;15(1):53-63.

2. Maia TV, Cooney RE, Peterson BS. The neural bases of obsessivecompulsive disorder in children and adults. Dev Psychopathol 2008;20(4):1251-83.

3. Monzani B, Rijsdijk F, Harris J, Mataix-Cols D. The structure of genetic and environmental risk factors for dimensional representations of DSM-5 obsessive-compulsive spectrum disorders. JAMA Psychiatry 2014;71(2):182-9.

4. Leo RJ, Regno PD. Atypical antipsychotic use in the treatment of psychosis in primary care. Prim Care Companion J Clin Psychiatry 2000;2(6):194-204.

5. Roopadevi H, Ramesh K, Nagabushan H. Pattern of psychotropic prescription in a tertiary care teaching hospital: A critical analysis. Asian J Pharm Clin Res 2015;8(5):252-5.

6. O'Brien A. Comparing the risk of tardive dyskinesia in older adults with first-generation and second-generation antipsychotics: A systematic review and meta-analysis. Int J Geriatr Psychiatry 2016;31:683-93.

7. Freedman R. Schizophrenia. N Engl J Med 2003;349(18):1738-49.

8. Miller R. Mechanisms of action of antipsychotic drugs of different classes, refractoriness to therapeutic effects of classical neuroleptics, and individual variation in sensitivity to their actions: Part II. Curr Neuropharmacol 2009;7(4):315-30.

9. Miller R. Mechanisms of action of antipsychotic drugs of different classes, refractoriness to therapeutic effects of classical neuroleptics, and individual variation in sensitivity to their actions: Part I. Curr Neuropharmacol 2009;7(4):302-14.

10. Peluso MJ, Lewis SW, Barnes TR, Jones PB. Extrapyramidal motor side-effects of first- and second-generation antipsychotic drugs. $\mathrm{Br} \mathrm{J}$ Psychiatry 2012;200(5):387-92.

11. Boyda HN, Procyshyn RM, Pang CC, Hawkes E, Wong D, Jin CH, et al. Metabolic side-effects of the novel second-generation antipsychotic drugs asenapine and iloperidone: A comparison with olanzapine. PLoS One 2013;8(1):e53459.

12. Parnetti L, Amici S, Lanari A, Gallai V. Pharmacological treatment of non-cognitive disturbances in dementia disorders. Mech Ageing Dev 2001;122(16):2063-9.

13. Bari A, Eagle DM, Mar AC, Robinson ES, Robbins TW. Dissociable effects of noradrenaline, dopamine, and serotonin uptake blockade on stop task performance in rats. Psychopharmacology (Berl) 2009;205(2):273-83.

14. Klimek V, Roberson G, Stockmeier CA, Ordway GA. Serotonin transporter and MAO-B levels in monoamine nuclei of the human brainstem are normal in major depression. J Psychiatr Res 2003;37(5):387-97.

15. Montoya A, Bruins R, Katzman MA, Blier P. The noradrenergic paradox: Implications in the management of depression and anxiety. Neuropsychiatr Dis Treat 2016;12:541-57.

16. Damsa C, Bumb A, Bianchi-Demicheli F, Vidailhet P, Sterck R, Andreoli A, et al. "Dopamine-dependent" side effects of selective serotonin reuptake inhibitors: A clinical review. J Clin Psychiatry 2004;65(8):1064-8

17. Gregorian RS, Golden KA, Bahce A, Goodman C, Kwong WJ, Khan ZM. Antidepressant-induced sexual dysfunction. Ann Pharmacother 2002;36(10):1577-89.

18. Cheng YL, Hu HY, Lin XH, Luo JC, Peng YL, Hou MC, et al. Use of SSRI, but not SNRI, increased upper and lower gastrointestinal bleeding: A nationwide population-based cohort study in Taiwan. Medicine (Baltimore) 2015;94(46):e2022.

19. Selective serotonin reuptake inhibitors in obsessive-compulsive disorder. Drug Ther Bull 1995;33(6):47-8

20. Pallanti S, Quercioli L. Treatment-refractory obsessive-compulsive disorder: Methodological issues, operational definitions and therapeutic lines. Progr Neuro-Psychopharmacol Biol Psychiatry 2006;30(3):400-12.

21. Pallanti S, Hollander E, Bienstock C, Koran L, Leckman J, Marazziti D, Pato M, et al. Treatment non-response in OCD: Methodological issues and operational definitions. Int $\mathrm{J}$ Neuropsychopharmacol 2002;5(2):181-91.

22. Bloch MH, Landeros-Weisenberger A, Kelmendi B, Coric V, Bracken MB, Leckman JF. A systematic review: Antipsychotic augmentation with treatment refractory obsessive-compulsive disorder. Mol Psychiatry 2006;11(7):622-32.

23. Skapinakis P, Papatheodorou T, Mavreas V. Antipsychotic augmentation of serotonergic antidepressants in treatment-resistant obsessive-compulsive disorder: A meta-analysis of the randomized controlled trials. Eur Neuropsychopharmacol 2007;17(2):79-93.

24. Fujita M, Verhoeff NP, Varrone A, Zoghbi SS, Baldwin RM, Jatlow PA, et al. Imaging extrastriatal dopamine $\mathrm{D}(2)$ receptor occupancy by endogenous dopamine in healthy humans. Eur J Pharmacol 2000;387(2):179-88.

25. Shende V, Sahane R, Lawar M, Hamdulay N, Langote H. Evaluation of anti-compulsive effect of ethanolic extract of clitoria ternatea in mice. Asian J Pharm Clin Res 2012;5 Suppl 3:120.

26. Kuroki T, Nagao N, Nakahara T. Neuropharmacology of secondgeneration antipsychotic drugs: A validity of the serotonin-dopamine hypothesis. Prog Brain Res 2008;172:199-212.

27. Divac N, Prostran M, Jakovcevski I, Cerovac N. Second-generation antipsychotics and extrapyramidal adverse effects. Biomed Res Int 2014;2014:656370

28. Landsheer JA, Smit JH, van Oppen P, van Balkom AJ. Assignment refusal and its relation to outcome in a randomized controlled trial comparing Cognitive Therapy and Fluvoxamine in treatmentresistant patients with obsessive compulsive disorder. Psychiatry Res 2015;226(1):198-203.

29. Bussing R, Reid AM, McNamara JP, Meyer JM, Guzick AG, Mason DM, et al. A pilot study of actigraphy as an objective measure of SSRI activation symptoms: Results from a randomized placebo controlled psychopharmacological treatment study. Psychiatry Res 2015;225(3):440-5.

30. Skarphedinsson G, Weidle B, Thomsen PH, Dahl K, Torp NC, Nissen JB, et al. Continued cognitive-behavior therapy versus sertraline for children and adolescents with obsessive-compulsive disorder that were non-responders to cognitive-behavior therapy: A randomized controlled trial. Eur Child Adolesc Psychiatry 2015;24(5):591-602.

31. Conelea CA, Walther MR, Freeman JB, Garcia AM, Sapyta J, Khanna M, et al. Tic-related obsessive-compulsive disorder (OCD): Phenomenology and treatment outcome in the Pediatric OCD Treatment Study II. J Am Acad Child Adolesc Psychiatry 2014;53(12):1308-16.

32. Giasuddin NA, Nahar JS, Morshed NM, Balhara YP, Sobhan MA. Efficacy of combination of fluoxetine and cognitive behavioral therapy and fluoxetine alone for the treatment of obsessive compulsive disorder. Pak J Pharm Sci 2013;26(1):95-8.

33. Ghaleiha A, Entezari N, Modabbernia A, Najand B, Askari N, Tabrizi M, et al. Memantine add-on in moderate to severe obsessivecompulsive disorder: Randomized double-blind placebo-controlled 
study. J Psychiatr Res 2013;47(2):175-80.

34. Humble MB, Uvnäs-Moberg K, Engström I, Bejerot S. Plasma oxytocin changes and anti-obsessive response during serotonin reuptake inhibitor treatment: A placebo controlled study. BMC Psychiatry 2013;13:344.

35. Jakubovski E, Diniz JB, Valerio C, Fossaluza V, Belotto-Silva C, Gorenstein $\mathrm{C}$, et al. Clinical predictors of long-term outcome in obsessive-compulsive disorder. Depress Anxiety 2013;30(8):763-72.

36. Bussing R, Murphy TK, Storch EA, McNamara JP, Reid AM, Garvan CW, et al. Psychometric properties of the treatment-emergent activation and suicidality assessment profile (TEASAP) in youth with OCD. Psychiatry Res 2013;205(3):253-61.

37. Hoexter MQ, Dougherty DD, Shavitt RG, D'Alcante CC, Duran FL, Lopes AC, et al. Differential prefrontal gray matter correlates of treatment response to fluoxetine or cognitive-behavioral therapy in obsessive-compulsive disorder. Eur Neuropsychopharmacol 2013;23(7):569-80

38. Sayyah M, Olapour A, Saeedabad YS, Yazdan Parast R, Malayeri A. Evaluation of oral zinc sulfate effect on obsessive-compulsive disorder: A randomized placebo-controlled clinical trial. Nutrition 2012;28(9):892-5

39. Van Balkom AJ, Emmelkamp PM, Eikelenboom M, Hoogendoorn AW, Smit JH, van Oppen P. Cognitive therapy versus fluvoxamine as a second-step treatment in obsessive-compulsive disorder nonresponsive to first-step behavior therapy. Psychother Psychosom 2012;81(6):366-74

40. Hoexter MQ, de Souza Duran FL, D’Alcante CC, Dougherty DD, Shavitt RG, Lopes AC, et al. Gray matter volumes in obsessivecompulsive disorder before and after fluoxetine or cognitive-behavior therapy: A randomized clinical trial. Neuropsychopharmacology 2012;37(3):734-45.

41. Borges CP, Meyer E, Ferrão YA, Souza FP, Sousa MB, Cordioli AV. Cognitive-behavioral group therapy versus sertraline for obsessivecompulsive disorder: Five-year follow-up. Psychother Psychosom 2011;80(4):249-50

42. Sayyah M, Boostani H, Pakseresht S, Malayeri A. A preliminary randomized double-blind clinical trial on the efficacy of celecoxib as an adjunct in the treatment of obsessive-compulsive disorder. Psychiatry Res 2011;189(3):403-6.

43. Garcia AM, Sapyta JJ, Moore PS, Freeman JB, Franklin ME, March JS, et al. Predictors and moderators of treatment outcome in the Pediatric Obsessive Compulsive Treatment Study (POTS I). J Am Acad Child Adolesc Psychiatry 2010;49(10):1024-33

44. Sayyah M, Boostani H, Pakseresht S, Malayeri A. Comparison of Silybum marianum (L.) Gaertn. with fluoxetine in the treatment of obsessive-compulsive disorder. Prog Neuropsychopharmacol Biol Psychiatry 2010;34(2):362-5.

45. Soltani F, Sayyah M, Feizy F, Malayeri A, Siahpoosh A, Motlagh I. A double-blind, placebo-controlled pilot study of ondansetron for patients with obsessive-compulsive disorder. Hum Psychopharmacol 2010;25(6):509-13

46. Matsunaga H, Nagata T, Hayashida K, Ohya K, Kiriike N, Stein DJ. A long-term trial of the effectiveness and safety of atypical antipsychotic agents in augmenting SSRI-refractory obsessive-compulsive disorder. J Clin Psychiatry 2009;70(6):863-8.

47. Alaghband-Rad J, Hakimshooshtary M. A randomized controlled clinical trial of citalopram versus fluoxetine in children and adolescents with obsessive-compulsive disorder (OCD). Eur Child Adolesc Psychiatry 2009;18(3):131-5.

48. Stein DJ, Carey PD, Lochner C, Seedat S, Fineberg N, Andersen EW. Escitalopram in obsessive-compulsive disorder: Response of symptom dimensions to pharmacotherapy. CNS Spectr 2008;13(6):492-8.

49. Onder E, Tural U, Gökbakan M. Does gabapentin lead to early symptom improvement in obsessive-compulsive disorder? Eur Arch Psychiatry Clin Neurosci 2008;258(6):319-23.

50. Denys D, Fineberg N, Carey PD, Stein DJ. Quetiapine addition in obsessive-compulsive disorder: Is treatment outcome affected by type and dose of serotonin reuptake inhibitors? Biol Psychiatry 2007;61(3):412-4

51. Khan MN, Hotiana UA, Ahmad S. Escitalopram in the treatment of obsessive-compulsive disorder: A double blind placebo control trial. J Ayub Med Coll Abbottabad 2007;19(4):58-63.

52. Stein DJ, Andersen EW, Overo KF. Response of symptom dimensions in obsessive-compulsive disorder to treatment with citalopram or placebo. Rev Bras Psiquiatr 2007;29(4):303-7.

53. Fineberg NA, Pampaloni I, Pallanti S, Ipser J, Stein DJ. Sustained response versus relapse: The pharmacotherapeutic goal for obsessive-compulsive disorder. Int Clin Psychopharmacol 2007;22(6):313-22

54. Sousa MB, Isolan LR, Oliveira RR, Manfro GG, Cordioli AV. A randomized clinical trial of cognitive-behavioral group therapy and sertraline in the treatment of obsessive-compulsive disorder. J Clin Psychiatry 2006;67(7):1133-9.

55. Ninan PT, Koran LM, Kiev A, Davidson JR, Rasmussen SA, Zajecka JM, et al. High-dose sertraline strategy for nonresponders to acute treatment for obsessive-compulsive disorder: A multicenter double-blind trial. J Clin Psychiatry 2006;67(1):15-22.

56. Denys D, Fluitman S, Kavelaars A, Heijnen C, Westenberg HG. Effects of paroxetine and venlafaxine on immune parameters in patients with obsessive compulsive disorder. Psychoneuroendocrinology 2006;31(3):355-60

57. van Oppen P, van Balkom AJ, de Haan E, van Dyck R. Cognitive therapy and exposure in vivo alone and in combination with fluvoxamine in obsessive-compulsive disorder: A 5-year follow-up. J Clin Psychiatry 2005;66(11):1415-22.

58. Hollander E, Kaplan A, Schmeidler J, Yang H, Li D, Koran LM, et al. Neurological soft signs as predictors of treatment response to selective serotonin reuptake inhibitors in obsessive-compulsive disorder. J Neuropsychiatry Clin Neurosci 2005;17(4):472-7.

59. Asbahr FR, Castillo AR, Ito LM, Latorre MR, Moreira MN, Lotufo-Neto F. Group cognitive-behavioral therapy versus sertraline for the treatment of children and adolescents with obsessive-compulsive disorder. J Am Acad Child Adolesc Psychiatry 2005;44(11):1128-36.

60. Tenneij NH, van Megen HJ, Denys DA, Westenberg HG. Behavior therapy augments response of patients with obsessivecompulsive disorder responding to drug treatment. J Clin Psychiatry 2005;66(9):1169-75

61. Nakatani E, Nakagawa A, Nakao T, Yoshizato C, Nabeyama M, Kudo A, et al. A randomized controlled trial of Japanese patients with obsessive-compulsive disorder - effectiveness of behavior therapy and fluvoxamine. Psychother Psychosom 2005;74(5):269-76.

62. Rufer M, Hand I, Alsleben H, Braatz A, Ortmann J, Katenkamp B, et al. Long-term course and outcome of obsessive-compulsive patients after cognitive-behavioral therapy in combination with either fluvoxamine or placebo: A 7-year follow-up of a randomized double-blind trial. Eur Arch Psychiatry Clin Neurosci 2005;255(2):121-8.

63. Pediatric OCD Treatment Study (POTS) Team. Cognitivebehavior therapy, sertraline, and their combination for children and adolescents with obsessive-compulsive disorder: The Pediatric OCD Treatment Study (POTS) randomized controlled trial. JAMA 2004;292(16):1969-76.

64. Geller DA, Wagner KD, Emslie G, Murphy T, Carpenter DJ, Wetherhold E, et al. Paroxetine treatment in children and adolescents with obsessive-compulsive disorder: A randomized, multicenter, double-blind, placebo-controlled trial. J Am Acad Child Adolesc Psychiatry 2004;43(11):1387-96.

65. Pallanti S, Quercioli L, Bruscoli M. Response acceleration with mirtazapine augmentation of citalopram in obsessive-compulsive disorder patients without comorbid depression: A pilot study. J Clin Psychiatry 2004;65(10):1394-9.

66. Kamijima K, Murasaki M, Asai M, Higuchi T, Nakajima T, Taga C, et al. Paroxetine in the treatment of obsessive-compulsive disorder: Randomized, double-blind, placebo-controlled study in Japanese patients. Psychiatry Clin Neurosci 2004;58(4):427-33.

67. Fux M, Benjamin J, Nemets B. A placebo-controlled cross-over trial of adjunctive EPA in OCD. J Psychiatr Res 2004;38(3):323-5.

68. Neziroglu F, Pinto A, Yaryura-Tobias JA, McKay D. Overvalued ideation as a predictor of fluvoxamine response in patients with obsessive-compulsive disorder. Psychiatry Res 2004;125(1):53-60.

69. Hollander E, Baldini Rossi N, Sood E, Pallanti S. Risperidone augmentation in treatment-resistant obsessive-compulsive disorder: A double-blind, placebo-controlled study. Int J Neuropsychopharmacol 2003;6(4):397-401.

70. Hollander E, Allen A, Steiner M, Wheadon DE, Oakes R, Burnham DB; Paroxetine OCD Study Group. Acute and long-term treatment and prevention of relapse of obsessive-compulsive disorder with paroxetine. J Clin Psychiatry 2003;64(9):1113-21.

71. Hollander E, Friedberg J, Wasserman S, Allen A, Birnbaum M, Koran LM. Venlafaxine in treatment-resistant obsessive-compulsive disorder. J Clin Psychiatry 2003;64(5):546-50

72. Denys D, van der Wee N, van Megen HJ, Westenberg HG. A double blind comparison of venlafaxine and paroxetine in obsessivecompulsive disorder. J Clin Psychopharmacol 2003;23(6):568-75.

73. Denys D, van Megen HJ, Westenberg HG. Emerging skin-picking 
behaviour after serotonin reuptake inhibitor-treatment in patients with obsessive-compulsive disorder: Possible mechanisms and implications for clinical care. J Psychopharmacol 2003;17(1):127-9.

74. Geller DA, Biederman J, Stewart SE, Mullin B, Farrell C, Wagner KD, et al. Impact of comorbidity on treatment response to paroxetine in pediatric obsessive-compulsive disorder: Is the use of exclusion criteria empirically supported in randomized clinical trials? J Child Adolesc Psychopharmacol 2003;13 Suppl 1:S19-29.

75. Hollander E, Koran LM, Goodman WK, Greist JH, Ninan PT, Yang H, et al. A double-blind, placebo-controlled study of the efficacy and safety of controlled-release fluvoxamine in patients with obsessivecompulsive disorder. J Clin Psychiatry 2003;64(6):640-7.

76. Liebowitz MR, Turner SM, Piacentini J, Beidel DC, Clarvit SR, Davies SO, et al. Fluoxetine in children and adolescents with OCD: A placebo-controlled trial. J Am Acad Child Adolesc Psychiatry 2002;41(12):1431-8.

77. Albert U, Aguglia E, Maina G, Bogetto F. Venlafaxine versus clomipramine in the treatment of obsessive-compulsive disorder: A preliminary single-blind, 12-week, controlled study. J Clin Psychiatry 2002;63(11):1004-9.

78. Romano S, Goodman W, Tamura R, Gonzales J. Long-term treatment of obsessive-compulsive disorder after an acute response: A comparison of fluoxetine versus placebo. J Clin Psychopharmacol 2001;21(1):46-52

79. Bergeron R, Ravindran AV, Chaput Y, Goldner E, Swinson R, van Ameringen MA, et al. Sertraline and fluoxetine treatment of obsessive-compulsive disorder: Results of a double-blind, 6-month treatment study. J Clin Psychopharmacol 2002;22(2):148-54.

80. Stein DJ, Montgomery SA, Kasper S, Tanghoj P. Predictors of response to pharmacotherapy with citalopram in obsessive-compulsive disorder. Int Clin Psychopharmacol 2001;16(6):357-61.

81. Eisen JL, Rasmussen SA, Phillips KA, Price LH, Davidson J, Lydiard RB, et al. Insight and treatment outcome in obsessivecompulsive disorder. Compr Psychiatry 2001;42(6):494-7.

82. Cook EH, Wagner KD, March JS, Biederman J, Landau P, Wolkow R, et al. Long-term sertraline treatment of children and adolescents with obsessive-compulsive disorder. J Am Acad Child Adolesc Psychiatry 2001;40(10):1175-81

83. Geller DA, Hoog SL, Heiligenstein JH, Ricardi RK, Tamura R, Kluszynski S, et al. Fluoxetine treatment for obsessive-compulsive disorder in children and adolescents: A placebo-controlled clinical trial. J Am Acad Child Adolesc Psychiatry 2001;40(7):773-9.

84. Humble M, Bejerot S, Bergqvist PB, Bengtsson F. Reactivity of serotonin in whole blood: Relationship with drug response in obsessive-compulsive disorder. Biol Psychiatry 2001;49(4):360-8.

85. Riddle MA, Reeve EA, Yaryura-Tobias JA, Yang HM, Claghorn JL, Gaffney G, et al. Fluvoxamine for children and adolescents with obsessive-compulsive disorder: A randomized, controlled, multicenter trial. J AmAcad Child Adolesc Psychiatry 2001;40(2):222-9.

86. Peter H, Tabrizian S, Hand I. Serum cholesterol in patients with obsessive compulsive disorder during treatment with behavior therapy and SSRI or placebo. Int J Psychiatry Med 2000;30(1):27-39.

87. Dannon PN, Sasson Y, Hirschmann S, Iancu I, Grunhaus LJ, Zohar J. Pindolol augmentation in treatment-resistant obsessive compulsive disorder: A double-blind placebo controlled trial. Eur Neuropsychopharmacol 2000;10(3):165-9.

88. Neziroglu F, Stevens KP, McKay D, Yaryura-Tobias JA. Predictive validity of the overvalued ideas scale: Outcome in obsessivecompulsive and body dysmorphic disorders. Behav Res Ther 2001;39(6):745-56.

89. Hoehn-Saric R, Ninan P, Black DW, Stahl S, Greist JH, Lydiard B, et al. Multicenter double-blind comparison of sertraline and desipramine for concurrent obsessive-compulsive and major depressive disorders. Arch Gen Psychiatry 2000;57(1):76-82.

90. Pallanti S, Quercioli L, Paiva RS, Koran LM. Citalopram for treatment-resistant obsessive-compulsive disorder. Eur Psychiatry 1999;14(2):101-6

91. Rosenberg DR, Stewart CM, Fitzgerald KD, Tawile V, Carroll E. Paroxetine open-label treatment of pediatric outpatients with obsessive-compulsive disorder. J Am Acad Child Adolesc Psychiatry 1999;38(9):1180-5

92. Zitterl W, Meszaros K, Hornik K, Twaroch T, Dossenbach M, Zitterl-Eglseer K, et al. Efficacy of fluoxetine in Austrian patients with obsessive-compulsive disorder. Wien Klin Wochenschr 1999;111(11):439-42.

93. Mundo E, Bareggi SR, Pirola R, Bellodi L. Effect of acute intravenous clomipramine and antiobsessional response to proserotonergic drugs: Is gender a predictive variable? Biol Psychiatry 1999;45(3):290-4.

94. van Balkom AJ, de Haan E, van Oppen P, Spinhoven P, Hoogduin KA, van Dyck R. Cognitive and behavioral therapies alone versus in combination with fluvoxamine in the treatment of obsessive compulsive disorder. J Nerv Ment Dis 1998;186(8):492-9.

95. Greenberg BD, Benjamin J, Martin JD, Keuler D, Huang SJ, Altemus $\mathrm{M}$, et al. Delayed obsessive-compulsive disorder symptom exacerbation after a single dose of a serotonin antagonist in fluoxetinetreated but not untreated patients. Psychopharmacology (Berl) 1998;140(4):434-44.

96. March JS, Biederman J, Wolkow R, Safferman A, Mardekian J, Cook EH, et al. Sertraline in children and adolescents with obsessivecompulsive disorder: A multicenter randomized controlled trial. JAMA 1998;280(20):1752-6.

97. Hohagen F, Winkelmann G, Rasche-Rüchle H, Hand I, König A, Münchau N, et al. Combination of behaviour therapy with fluvoxamine in comparison with behaviour therapy and placebo. Results of a multicentre study. Br J Psychiatry Suppl 1998;71-8.

98. Ackerman DL, Greenland S, Bystritsky A. Clinical characteristics of response to fluoxetine treatment of obsessive-compulsive disorder. J Clin Psychopharmacol 1998;18(3):185-92.

99. Mundo E, Bareggi SR, Pirola R, Bellodi L, Smeraldi E. Long-term pharmacotherapy of obsessive-compulsive disorder: A double-blind controlled study. J Clin Psychopharmacol 1997;17(1):4-10.

100. Milanfranchi A, Ravagli S, Lensi P, Marazziti D, Cassano GB. A double-blind study of fluvoxamine and clomipramine in the treatment of obsessive-compulsive disorder. Int Clin Psychopharmacol 1997;12(3):131-6

101. López-Ibor JJ Jr, Saiz J, Cottraux J, Note I, Viñas R, Bourgeois M, et al. Double-blind comparison of fluoxetine versus clomipramine in the treatment of obsessive compulsive disorder. Eur Neuropsychopharmacol 1996;6(2):111-8.

102. Dell'Osso B, Altamura AC, Mundo E, Marazziti D, Hollander E. Diagnosis and treatment of obsessive-compulsive disorder and related disorders. Int J Clin Pract 2007;61(1):98-104.

103. Irons J. Fluvoxamine in the treatment of anxiety disorders. Neuropsychiatr Dis Treat 2005;1(4):289-99.

104. Pittenger C, Bloch MH, Wasylink S, Billingslea E, Simpson R, Jakubovski E, et al. Riluzole augmentation in treatment-refractory obsessive-compulsive disorder: AA pilot randomized placebocontrolled trial. J Clin Psychiatry 2015;76(8):1075-84

105. Foa EB, Simpson HB, Rosenfield D, Liebowitz MR, Cahill SP, Huppert JD, et al. Six-month outcomes from a randomized trial augmenting serotonin reuptake inhibitors with exposure and response prevention or risperidone in adults with obsessive-compulsive disorder. J Clin Psychiatry 2015;76(4):440-6.

106. Simpson HB, Foa EB, Liebowitz MR, Huppert JD, Cahill S, Maher MJ, et al. Cognitive-behavioral therapy vs risperidone for augmenting serotonin reuptake inhibitors in obsessive-compulsive disorder: A randomized clinical trial. JAMA Psychiatry 2013;70(11):1190-9.

107. Storch EA, Goddard AW, Grant JE, De Nadai AS, Goodman WK, Mutch PJ, et al. Double-blind, placebo-controlled, pilot trial of paliperidone augmentation in serotonin reuptake inhibitor-resistant obsessive-compulsive disorder. J Clin Psychiatry 2013;74(6):e527-32.

108. Sayyah M, Sayyah M, Boostani H, Ghaffari SM, Hoseini A. Effects of aripiprazole augmentation in treatment-resistant obsessivecompulsive disorder (a double blind clinical trial). Depress Anxiety 2012;29(10):850-4

109. Muscatello MR, Bruno A, Pandolfo G, Micò U, Scimeca G, Romeo VM, et al. Effect of aripiprazole augmentation of serotonin reuptake inhibitors or clomipramine in treatment-resistant obsessivecompulsive disorder: A double-blind, placebo-controlled study. J Clin Psychopharmacol 2011;31(2):174-9.

110. Selvi Y, Atli A, Aydin A, Besiroglu L, Ozdemir P, Ozdemir O. The comparison of aripiprazole and risperidone augmentation in selective serotonin reuptake inhibitor-refractory obsessive-compulsive disorder: A single-blind, randomised study. Hum Psychopharmacol 2011;26(1):51-7.

111. Diniz JB, Shavitt RG, Pereira CA, Hounie AG, Pimentel I, Koran LM, et al. Quetiapine versus clomipramine in the augmentation of selective serotonin reuptake inhibitors for the treatment of obsessive-compulsive disorder: A randomized, open-label trial. J Psychopharmacol 2010;24(3):297-307.

112. Kordon A, Wahl K, Koch N, Zurowski B, Anlauf M, Vielhaber K, et al. Quetiapine addition to serotonin reuptake inhibitors in patients with 
severe obsessive-compulsive disorder: A double-blind, randomized, placebo-controlled study. J Clin Psychopharmacol 2008;28(5):550-4.

113. de Geus F, Denys D, Westenberg HG. Effects of quetiapine on cognitive functioning in obsessive-compulsive disorder. Int Clin Psychopharmacol 2007;22(2):77-84.

114. Buchsbaum MS, Hollander E, Pallanti S, Baldini Rossi N, Platholi J, Newmark $\mathrm{R}$, et al. Positron emission tomography imaging of risperidone augmentation in serotonin reuptake inhibitor-refractory patients. Neuropsychobiology 2006;53(3):157-68.

115. Erzegovesi S, Guglielmo E, Siliprandi F, Bellodi L. Low-dose risperidone augmentation of fluvoxamine treatment in obsessivecompulsive disorder: A double-blind, placebo-controlled study. Eur Neuropsychopharmacol 2005;15(1):69-74

116. Li X, May RS, Tolbert LC, Jackson WT, Flournoy JM, Baxter LR. Risperidone and haloperidol augmentation of serotonin reuptake inhibitors in refractory obsessive-compulsive disorder: A crossover study. J Clin Psychiatry 2005;66(6):736-43.

117. Bogan AM, Koran LM, Chuong HW, Vapnik T, Bystritsky A. Quetiapine augmentation in obsessive-compulsive disorder resistant to serotonin reuptake inhibitors: An open-label study. J Clin Psychiatry 2005;66(1):73-9.

118. Carey PD, Vythilingum B, Seedat S, Muller JE, van Ameringen M, Stein DJ. Quetiapine augmentation of SRIs in treatment refractory obsessive-compulsive disorder: A double-blind, randomised, placebocontrolled study [ISRCTN83050762]. BMC Psychiatry 2005;5:5.

119. Denys D, de Geus F, van Megen HJ, Westenberg HG. A double-blind, randomized, placebo-controlled trial of quetiapine addition in patients with obsessive-compulsive disorder refractory to serotonin reuptake inhibitors. J Clin Psychiatry 2004;65(8):1040-8.

120. Bystritsky A, Ackerman DL, Rosen RM, Vapnik T, Gorbis E, Maidment KM, et al. Augmentation of serotonin reuptake inhibitors in refractory obsessive-compulsive disorder using adjunctive olanzapine: A placebo-controlled trial. J Clin Psychiatry 2004;65(4):565-8.

121. D’Amico G, Cedro C, Muscatello MR, Pandolfo G, Di Rosa AE, Zoccali R, et al. Olanzapine augmentation of paroxetine-refractory obsessive-compulsive disorder. Progr Neuro Psychopharmacol Biol Psychiatry 2003;27(4):619-23.

122. Denys D, van Megen $H$, Westenberg $H$. Quetiapine addition to serotonin reuptake inhibitor treatment in patients with treatmentrefractory obsessive-compulsive disorder: An open-label study. J Clin Psychiatry 2002;63(8):700-3.

123. Atmaca M, Kuloglu M, Tezcan E, Gecici O. Quetiapine augmentation in patients with treatment resistant obsessive-compulsive disorder: A single-blind, placebo-controlled study. Int Clin Psychopharmacol 2002;17(3):115-9.
124. Pfanner C, Marazziti D, Dell'Osso L, Presta S, Gemignani A, Milanfranchi A, et al. Risperidone augmentation in refractory obsessive-compulsive disorder: An open-label study. Int Clin Psychopharmacol 2000;15(5):297-301.

125. McDougle CJ, Epperson CN, Pelton GH, Wasylink S, Price LH. A double-blind, placebo-controlled study of risperidone addition in serotonin reuptake inhibitor-refractory obsessive-compulsive disorder. Arch Gen Psychiatry 2000;57(8):794-801.

126. Weiss EL, Potenza MN, McDougle CJ, Epperson CN. Olanzapine addition in obsessive-compulsive disorder refractory to selective serotonin reuptake inhibitors: An open-label case series. J Clin Psychiatry 1999;60(8):524-7.

127. Vulink NC, Westenberg HG, van Nieuwerburgh F, Deforce D, Fluitman SB, Meinardi JS, et al. Catechol-O-methyltranferase gene expression is associated with response to citalopram in obsessivecompulsive disorder. Int J Psychiatry Clin Pract 2012;16(4):277-83.

128. Diniz JB, Shavitt RG, Fossaluza V, Koran L, Pereira CA, Miguel EC. A double-blind, randomized, controlled trial of fluoxetine plus quetiapine or clomipramine versus fluoxetine plus placebo for obsessive-compulsive disorder. J Clin Psychopharmacol 2011;31(6):763-8

129. Simpson HB, Foa EB, Liebowitz MR, Ledley DR, Huppert JD, Cahill $\mathrm{S}$, et al. A randomized, controlled trial of cognitive-behavioral therapy for augmenting pharmacotherapy in obsessive-compulsive disorder. Am J Psychiatry 2008;165(5):621-30.

130. Shapira NA, Ward HE, Mandoki M, Murphy TK, Yang MC, Blier P, et al. A double-blind, placebo-controlled trial of olanzapine addition in fluoxetine-refractory obsessive-compulsive disorder. Biol Psychiatry 2004;55(5):553-5.

131. Stein DJ, Fineberg NA, Bienvenu OJ, Denys D, Lochner C, Nestadt G, et al. Should OCD be classified as an anxiety disorder in DSM-V? Depress Anxiety 2010;27(6):495-506.

132. Harding KJ, Skritskaya N, Doherty E, Fallon BA. Advances in understanding illness anxiety. Curr Psychiatry Rep 2008;10(4):311-7.

133. Franz AP, Paim M, Araujo RM, Rosa Vde O, Barbosa IM, Blaya C, et al. Treating refractory obsessive-compulsive disorder: What to do when conventional treatment fails? Trends Psychiatry Psychother 2013;35(1):24-35

134. Veale D, Miles S, Smallcombe N, Ghezai H, Goldacre B, Hodsoll J. Atypical antipsychotic augmentation in SSRI treatment refractory obsessive-compulsive disorder: A systematic review and metaanalysis. BMC Psychiatry 2014;14:317.

135. Arumugham SS, Reddy JY. Augmentation strategies in obsessivecompulsive disorder. Expert Rev Neurother 2013;13(2):187-202. 